\title{
ACOUSTIC EMISSION MODEL OF FATIGUE CRACK IN LOW-CARBON STEEL
}

\author{
BARAT V. A, MARCHENKOV A. YU, ELIZAROV S. V. \& BARDAKOV V. V
}

National Research University Moscow Power Engineering Institute, Moscow, Russian Federation

\begin{abstract}
One of the problems of the acoustic emission $(A E)$ testing method is the parametric uncertainty of defect models. In this work, an empirical model of a fatigue crack is considered, which allows establishing quantitative relations between the parameters of a fatigue crack and $A E$ data. The ambiguity in the interpretation of $A E$ data is explained by the inhomogeneous stress-strain state of the material.
\end{abstract}

KEYWORDS: Acoustic Emission, Fatigue Crack \& Model of Acoustic Emission Source

Received: Aug 10, 2019; Accepted: Aug 30, 2019; Published: Nov 04, 2019; Paper Id.: IJMPERDDEC201937

\section{INTRODUCTION}

Acoustic emission (AE) is a physical phenomenon associated with the emission of elastic waves by an object under test during nonlinear transformations of its structure. AE sources are dynamic processes associated with damaging of the solid structure at the micro, meso- and macro- levels.

$\mathrm{AE}$ sources at the micro level are dislocation movements. It has been experimentally and theoretically shown that $\mathrm{AE}$ is not a process associated with a single elementary dislocation act [1], but is determined by a collective process in which at least $10^{3}$ dislocations participate. Most likely, the process of emission of an acoustic signal occurs when the dislocation ensemble reaches the external surface of the crystal [2-4]. A large number of works published both in Russia [5-8] and in foreign publications [9-11] are devoted to the study of AE depending on the stage of plastic deformation. In these works, it is noted that the activity of AE coincides with the density of mobile dislocations during plastic deformation.

During deformation at the mesoscale, there is a similarity between the cumulative distributions of microcrack parameters and the parameters of AE impulse distributions. It was shown in [12] that at the initial stages of specimen deformation, the cumulative distribution of microcrack lengths and the distribution of AE impulse amplitudes correspond to an exponential law. The consistency of the distributions of microcrack lengths with the distributions of AE impulse parameters has been noted by many researchers; it was shown in [13] that the amplitude of the AE impulse is related to the size of the microcrack.

In the case of a crack, $\mathrm{AE}$ sources are the processes of plastic deformation at the tip of the crack and local discontinuities, due to which the formation of the crack surface occurs. The most common model of a crack as an AE source is the model of $\mathrm{H}$. Dunegan, which establishes a power-law relationship between the total number of AE impulses and crack length. The Palmer and Hild model, which allows one to relate the number of AE pulses with the applied mechanical stress $\sigma$ and crack length $l$, is also quite common. J. Holt and D. Godlart [3] proposed an AE model for a fatigue crack, based on which it is possible to determine the number of AE impulses emitted by a fatigue crack, depending on how much the load applied to the object exceeds the load applied at the stage of fatigue crack growing. 
Models of AE sources are well studied theoretically and confirmed experimentally; however, all of them have a significant drawback - parametric uncertainty. The functional dependence between the parameters characterizing the damage to the material is established up to parameters that depend on the thickness of the material, on its mechanical properties, as well as on the properties of the local stress-strain state, which cannot be known a priori. It is the parametric uncertainty of the models of AE sources that often leads to unreliable results of AE testing. Classical source models do not allow to quantitatively estimating AE parameters, such as activity, impulse amplitude, and total AE count.

Current paper considers empirical model of a fatigue crack in low-carbon steel 09G2S and quantitative values of $\mathrm{AE}$ parameters analysis. Correlation between the AE data and the dimensions and configuration of the plastic deformation zone at the crack tip is investigated.

\section{MATERIALS AND RESEARCH METHODS}

As the studied material, we chose the common structural low-carbon steel of pearlite class 09G2S. According to [14, 15] tanks, pipelines and vessels operating under pressure can be made of steel 09G2S. The chemical composition of steel is given in Table 1.

Table 1: The Chemical Composition of Steel 09G2S, \%

\begin{tabular}{|c|c|c|c|c|c|c|c|c|c|c|}
\hline $\mathbf{C}$ & $\mathbf{S i}$ & $\mathbf{M n}$ & $\mathbf{N i}$ & $\mathbf{S}$ & $\mathbf{P}$ & $\mathbf{C r}$ & $\mathbf{N}$ & $\mathbf{C u}$ & $\mathbf{A s}$ & $\mathbf{F e}$ \\
\hline$<0,12$ & $0,5-0,8$ & $1,3-1,7$ & $<0,3$ & $<0,04$ & $<0,035$ & $<0,3$ & $<0,008$ & $<0,3$ & $<0,08$ & $\sim 96-97$ \\
\hline
\end{tabular}

Samples for mechanical tests were made of sheet metal with a thickness of 3 and $5 \mathrm{~mm}$ using laser cutting. For testing, a series of samples of a rectangular shape with an edge notch was produced.

The thicknesses of the samples were 3 and $5 \mathrm{~mm}$, and the width $b=50 \mathrm{~mm}$. The size of the working zone $L$, based on the condition $L \geq 2 b$ and taking into account the area of the grips, was chosen equal to $350 \mathrm{~mm}$. The notch width was $4 \mathrm{~mm}$, the opening angle $\Theta=45^{\circ}$, and the notch depth $h=10 \mathrm{~mm}$. Additional changes were made to the sample geometry - 4 through holes with a diameter of $12 \mathrm{~mm}$ were added. Through holes are required to fix the damping pads, which reduce the level of acoustic noise generated by the loading machine (Figure 1).

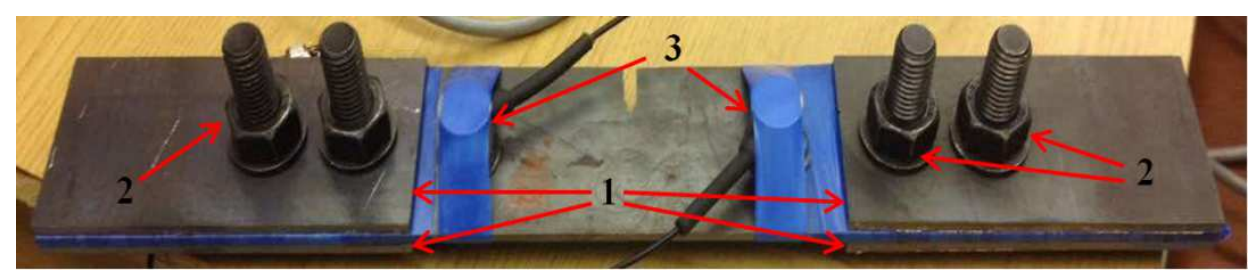

1- Damping Pads, 2 - Fixing Bolts, 3 - AE Sensors

Figure 1: Samples for Mechanical Tests.

The tests were carried out using an INSTRON 8801 servo-hydraulic testing machine (Figure 2). During the tests, the registration of acoustic signals was carried out using the INTERUNIS-IT industrial system A-Line 32D. The measuring path included the Global Testresonant sensors GT200 and the PAEF-014 electric signal pre-amplifiers with a gain of 26 $\mathrm{dB}$. The installation of the AE sensors on the surface of the object is carried out through the contact medium. AE sensors were fixed on the sample surface with insulating tape. According to the values of the maximum noise level and the recommendations in the regulatory document PB 03-593-03, the discrimination threshold for acoustic signals was chosen equal to $40 \mathrm{~dB}$. 


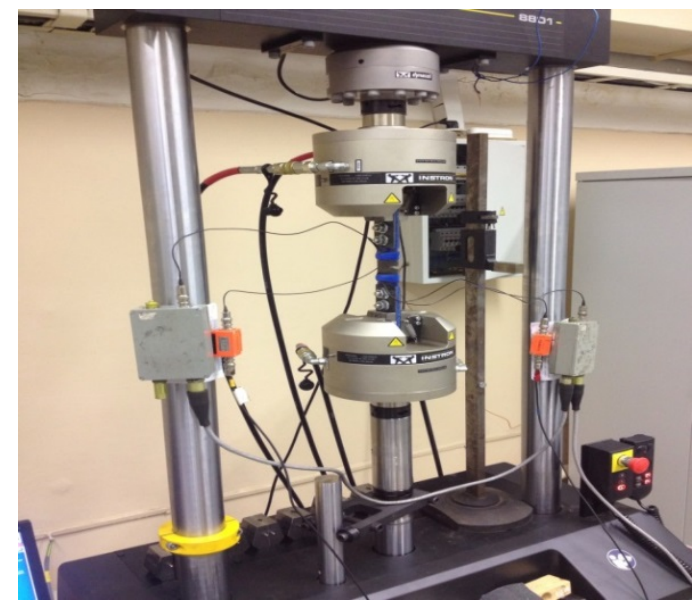

Figure 2: INSTRON 8801 Testing Machine during Cyclic Loading of a Sample with Applied AE Method.

The tests were carried out according to a scheme consisting of two consecutive stages. At the first stage, a side-cut specimen was loaded with triangular zero - starting cycles (cycle asymmetry coefficient $\mathrm{R}=0$ ) with a frequency $f=5 \mathrm{~Hz}$ and a maximum cycle stress of $\sigma_{\max }=210 \mathrm{MPa}$, which approximately corresponds to $70 \%$ of the yield strength $\sigma_{\mathrm{T}}=305$ $\mathrm{MPa}$ of the investigated metal. The number of loading cycles was chosen in such a way that the fatigue crack developing under cyclic loading in the lateral notch region would reach a certain length. Then, the fatigue cracked specimen was loaded according to the scheme adopted during industrial AE control, in accordance with industrial safety rules PB-593-03 [16], to a stress $25 \%$ higher than the stress $\sigma_{\max }$ at which the defect was grown. The loading scheme is shown in Figure 3 (blue graph).

Two triangular cycles at the beginning of loading are necessary to equalize the internal mechanical stresses that arose as a result of preliminary cycling of the samples and when placing them in a snap. Acoustic activity that occurs during preloading, as a rule, is not associated with the processes of destruction of the material, and is not taken into account. The main loading stage begins with two hundredths of a second and consists of four main stages - in the first, the load varies from 0 to $50 \% \sigma_{\max }$, in the second from 50 to $75 \% \sigma_{\max }$, in the third from 75 to $100 \% \sigma_{\max }$. The fourth stage is carried out with a stress excess of $\sigma_{\max }$ by $25 \%$. In each experiment, the fatigue crack lengths are determined twice before and after loading.

In the process of loading, parametric information was recorded - the value of the loading force and the position of the active lower beam, the parameters of the AE impulses and the primary information - waveforms of the signals. The following is a typical example of AE data recorded during an experiment. Figure 3 a shows the dependence of the total number of AE impulses on time and the parametric graph is the dependence of the loading force on time. 


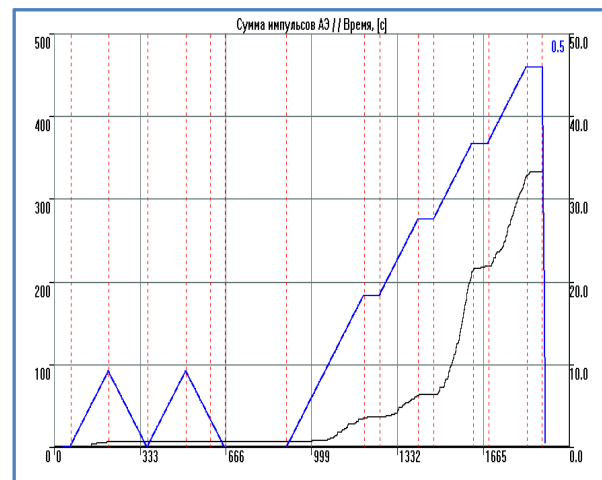

(a)

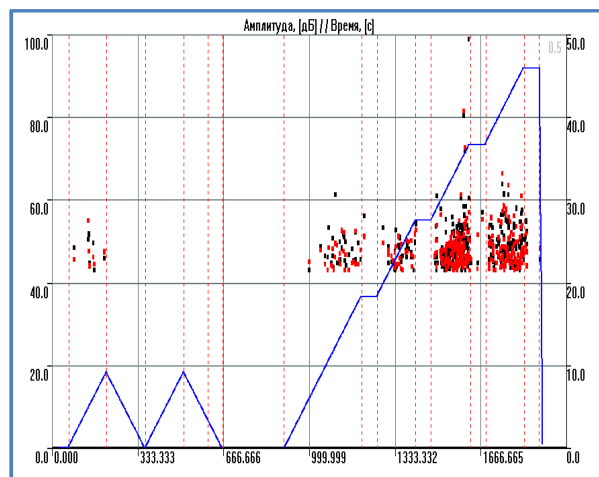

(b)

Figure 3: (a) Dependence of the Number of AE Pulses on Time (Black Graph), Dependence of the Loading Force on Time (Blue Graph) (b) Amplitude of AE Pulses Versus Time.

In the preloading area, the acoustic activity of the defect is negligible. At the main loading stage, the accumulation of $\mathrm{AE}$ pulses occurs with increasing load, and with increasing load above $0.75 P_{\text {max }}$, the dependence of the number of $\mathrm{AE}$ pulses on time has a pronounced power-law character.

Figure $3 b$ shows the time dependence of the AE pulses amplitudes. A typical range of amplitude values is from a threshold value to $65 \mathrm{~dB}$. As the load increases, an increasing trend in the values of AE impulse amplitudes is observed. The group of pulses with high amplitudes from 70 to $100 \mathrm{~dB}$, which was recorded at the third stage of loading, can be associated with the destruction of brittle non-metallic inclusions.

When analyzing AE data, filtering by location results is used to reduce the influence of noise from loading device — only AE pulses whose arrival time difference corresponds to the location of the fatigue crack are analyzed. The result of the location of the defect is shown in Figure 4 - the histogram corresponds to the number of AE events emitted at a point with a specific coordinate.

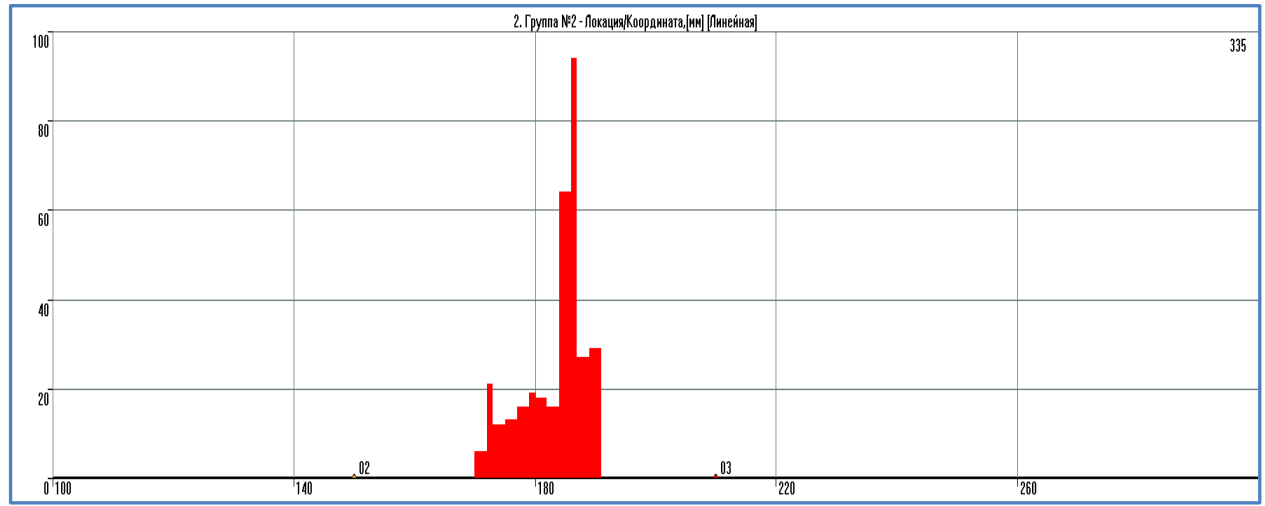

Figure 4: Location Result of the Fatigue Crack.

The maximum value in histogram is reached at the defect location point, and the spread of values along the abscissa axis of no more than $25 \mathrm{~mm}$ can be explained by the error in determining the difference in the arrival times of $\mathrm{AE}$ pulses, when AE sensors are placed closely.

A total of 53 samples were tested, 20 with $5 \mathrm{~mm}$ thick, 33 with $3 \mathrm{~mm}$ thick. Fatigue cracks were grown in the samples, the lengths of which varied from 15.5 to $28 \mathrm{~mm}$. The characteristics of fatigue cracks in obtained sample are shown in Table 2. 
Table 2: Characteristics of Fatigue Cracks

\begin{tabular}{|c|c|c|}
\hline & Crack Length, mm & Number of Samples \\
\hline 1 & from15,4to17,5 & 14 \\
\hline 2 & from17,5 to19,0 & 18 \\
\hline 3 & from19,0 to25,0 & 16 \\
\hline 4 & $>25,0$ & 5 \\
\hline
\end{tabular}

\section{DATA PROCESSING AND ANALYSIS}

\subsection{The Study of the Dynamics of the AE Impulses Accumulation}

The number of pulses $\mathrm{N}$ emitted during loading of samples with fatigue cracks turned out to be different even for cracks of approximately equal length. Figure 5 shows the correlation plane for the crack length $l$ and the number of emitted AE impulses. The randomness of the markers locations shows the absence of a correlation between these parameters. Considering that the parameter $\mathrm{N}$ is related to the volume of the plastic deformation zone at the crack tip, the scatter of this parameter can be explained by the difference in the volumes of plastic deformation for cracks with similar length.

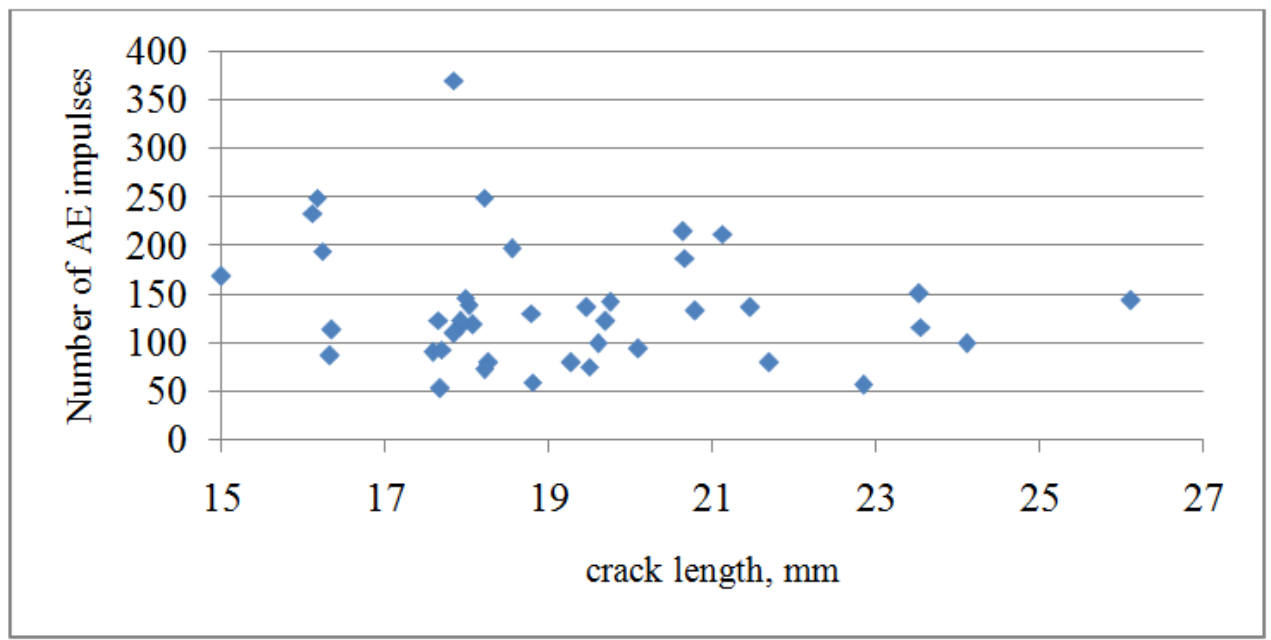

Figure 5: Correlation Plane for the Crack Length and the Number of Emitted AE Pulses.

Of interest is the fact that, the statistical distribution of the parameter $\mathrm{N}$ reliably corresponds to the log-normal law. Table 3 shows the results of checking the $\chi^{2}$ agreement criterion on data compliance with a log-normal distribution. The value of $p=0.48$ was about 10 times higher than the minimum level of confidence $\alpha=0.05$, which indicates a high reliability of the obtained results.

Table 3: The Results of Checking the Compliance of $\mathbf{N}$ - Distribution to Log-Normal Law

\begin{tabular}{|c|c|c|}
\hline$\chi^{2}$ Value & $\mathbf{p}-$ Value & Degrees of Freedom \\
\hline 2,46 & 0,48 & 3 \\
\hline
\end{tabular}

Figure 6 shows the empirical and cumulative distribution of the number of impulses N. The histogram corresponds to the empirical distribution, the theoretical dependence for the log normal distribution is shown in red. 


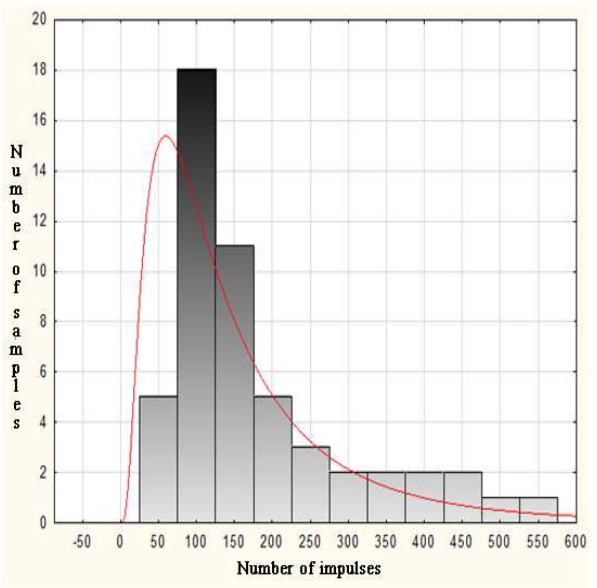

(a)

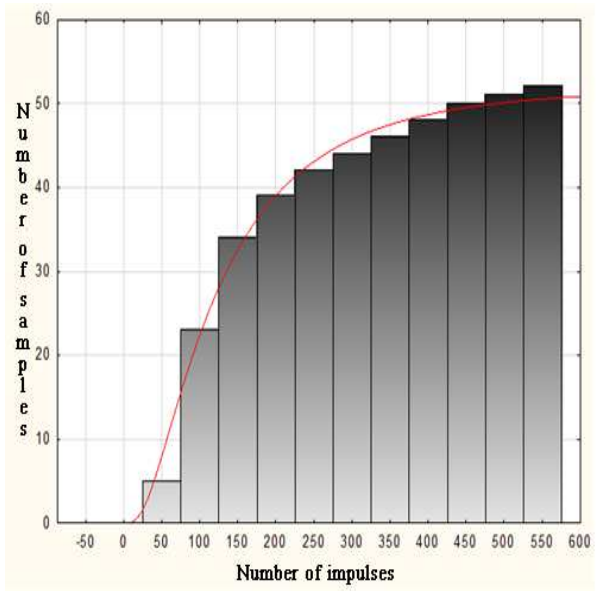

(b)

Figure 6: Differential (a) and Cumulative (b) Distribution of the Number of Impulses N.

In fundamental works on $\mathrm{AE}$, the relationship between the number of $\mathrm{AE}$ pulses $N$ and the increment of the volume of the plastic deformation zone $V_{p}$ at the crack tip during loading of the material is proved:

$$
N=D \cdot V_{p}
$$

It is noted that the proportionality coefficient $D$ depends on the magnitude of the load, temperature, thickness of the sample and the microstructure of the material.

From the theory of fracture mechanics, A. Griffiths's approach [17] to the process of the development of cracks in materials is known. According to the energy criterion proposed by him, for the propagation of a crack in a sufficiently brittle material, it is necessary to provide energy to its tip. Later, based on the ideas of Griffiths, the force criterion of J. Irwin and the concept of quasi-brittle fracture of E. Orovan and J. Irwin were formed, which became applicable to real metallic materials with some plasticity margin. An analysis of the Griffiths-Orovan-Irwin concept [18] suggests that a developed zone of plastic deformation is formed near the crack tip, the extent of which depends on the crack length and material properties and can reach up to $20 \%$ of its length. At the same time, in metallic materials, the process of crack development and the formation of a plastic deformation zone near its tipis also determined by the features of the real atomic-crystalline structure and microstructure of materials [19]. For the studied samples made of sheet steel 09G2S, the influence of these factors on the development of the plastic deformation zone and fracture is especially great.

First of all, this is due to the two-phase ferrite-pearlite structure of steel. The steel structure is relatively finegrained, with uneven microstructure along the sheet thickness - the metal microstructure near the surface is homogeneous, and the grain shape is close to equiaxial, while perlite grains elongated in the rolling direction are observed in the central part of the sheet. Obviously, such a metal structure, formed due to the peculiarities of the technology for producing a semifinished product, can lead to an increase in the spread in the intensity of crack growth over time, as well as from sample to sample. In addition, metallographic studies revealed non-metallic inclusions of various types - large single inclusions were found, as well as sulfide inclusions elongated in the rolling direction and being stress concentrators, which significantly reduce the energy needed for nucleation of defects such as cracks.

With insignificant differences in the size of the initial crack and the same loading parameters during the growth of the crack, the plastic deformation zones at the crack tip have different volumes, which is due to the peculiarities of the metal microstructure near the crack tip. Figure 7 shows microphotographs of crack tips obtained on the same samples 
tested under the same loading conditions. Qualitatively, the volume of the plastic deformation zone can be estimated from microphotographs of the sample regions near the crack tip in various sections.

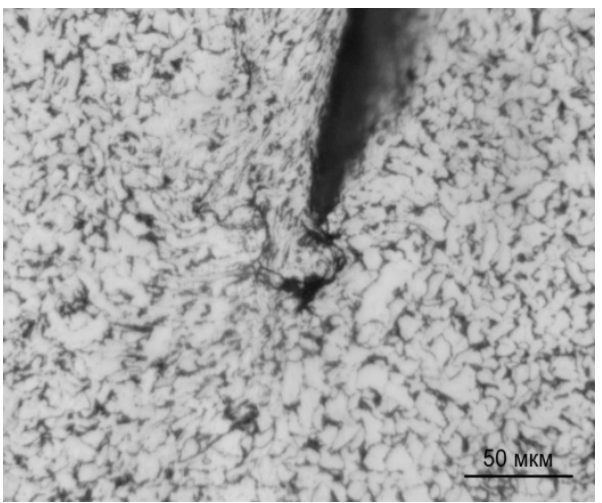

(a)

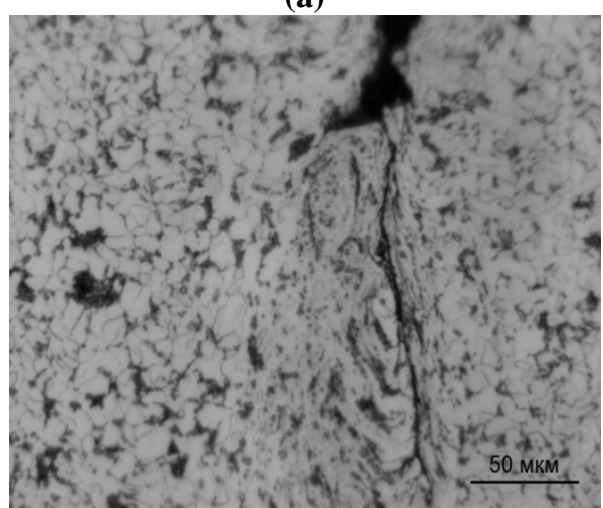

(c)

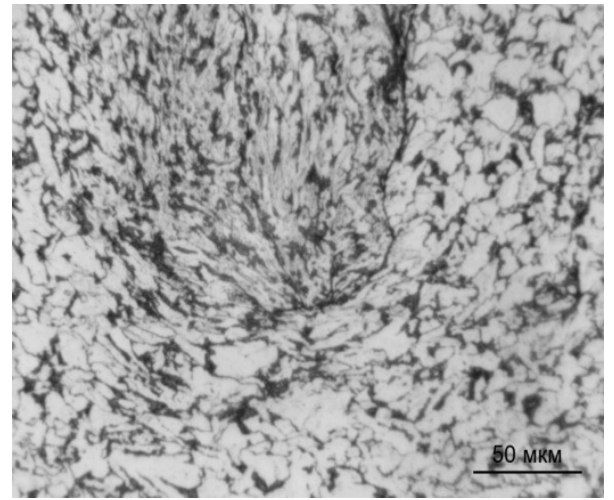

(b)

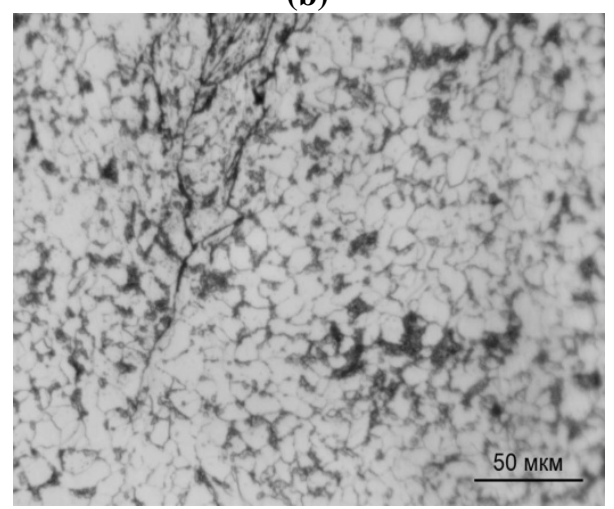

(d)

Figure 7: Cracks tips in Samples RB14 (a, b) and RB23 (c, d) a, c - near the Surface; b, $\mathrm{d}$ - a cut in Thickness of $0.25 \mathrm{~mm}$.

Obviously, for the samples studied, there is a difference in the nature of the zone of plastic deformation in the metal near the crack tip - the zones have different sizes and stress-strain state. When comparing the volume of the plastic deformation zone and the number of emitted AE impulses, a direct proportionality of these parameters was established. When testing samples for which, according to the results of metallographic studies, a larger volume of plastic deformation was established, a greater number of $\mathrm{AE}$ impulses were recorded. So, for example, $82 \mathrm{AE}$ impulses were recorded for the RB14 sample during the test cycle, 233 impulses were recorded for the RB23 sample, while the fatigue crack lengths in these samples were close to each other and equal to 18.15 and $18.26 \mathrm{~mm}$, respectively.

\subsection{Analysis of the Dynamics of AE Impulses Accumulation}

As a result of the analysis of experimental data, it was found that the number of AE impulses $\mathrm{N}$ varies depending on the applied load according to a power law, which corresponds to the model of H. Dannegan [3]

$$
N \sim P^{\alpha},
$$

Where, $N$ is the number of AE impulses, $P$ is the load value, $\alpha$ is a power index depending on stress and crack length.

For cracks with a length of less than $16 \mathrm{~mm}$, the characteristic value of the index $\alpha$ does not exceed 1 . Figure $8 \mathrm{a}$ shows the dependences of the sum of impulses on the loading force for defects with 16 and $15.8 \mathrm{~mm}$ lengths in a sample 
with thickness of $3 \mathrm{~mm}$. They have a nonlinear power-law character with different indexes of power, from 0.8 to 1.1 in different loading areas.

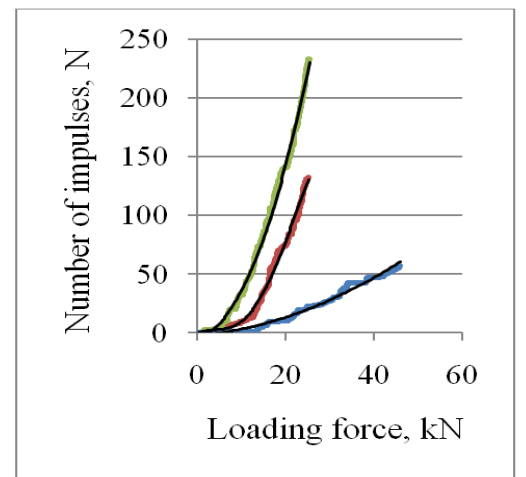

(a)

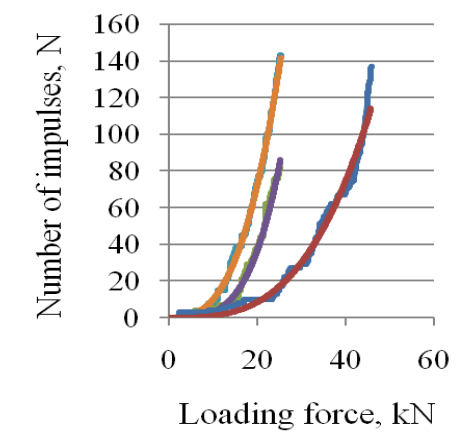

(b)

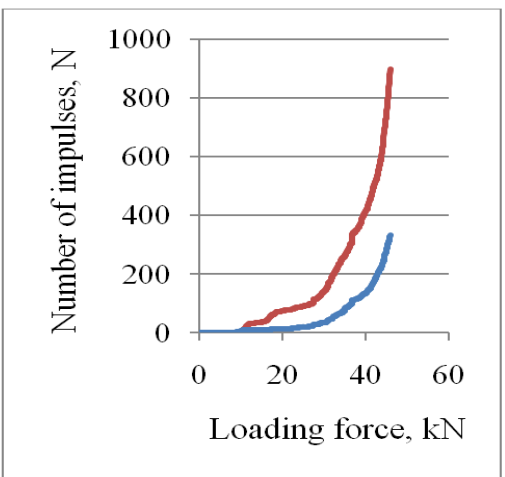

(c)

Figure 8: Dependence of the Number of AE Impulses on the Load (a) - for Fatigue Cracks with a Length Less than 16 mm; (b)- for Fatigue Cracks with a Length from 16 to 20 mm; (c) - for Fatigue Cracks with a Length from 20 to $25 \mathrm{~mm}$.

For cracks of greater length, the dependence $N(P)$ has a pronounced power-law character. For cracks with a length from 16 to $20 \mathrm{~mm}$, the power-law index lies in the range from 2.5 to 4 . Examples of such dependences are shown in Figure 8b. Three power dependences correspond to cracks with length of 17.4, 18.9 and 19.8mm. For cracks more than 20 mm and less than $25 \mathrm{~mm}$ long, the power index $\alpha$ reaches 6-8 (Figure 8c). For specimens with critical fatigue cracks that broke under loading, the dependence $N(P)$ changes its character and corresponds to an exponential law.

The regression dependence of the power index $\alpha$ on the crack length is shown in Figure 9, the model can be written as (5)

$$
\alpha=0,36 l-3,83
$$

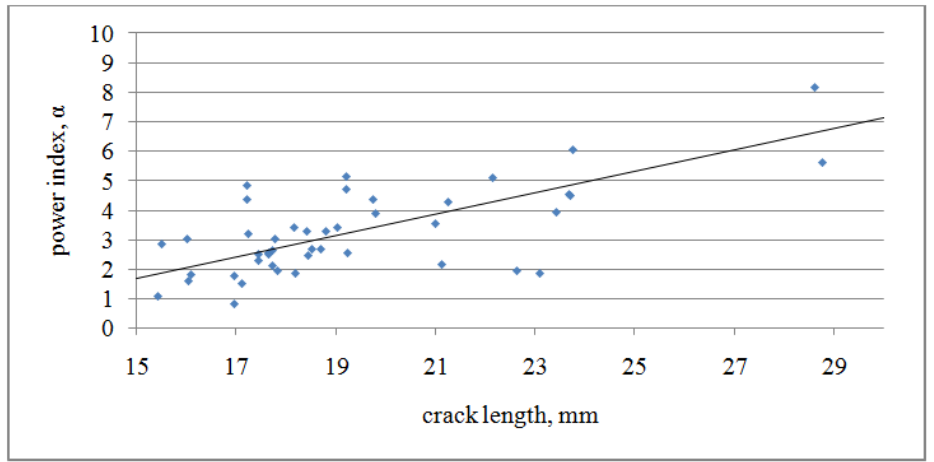

Figure 9: The Regression Dependence of the Power Index $\alpha$ on the Crack Length.

Despite the high value of the correlation coefficient of the crack length and $\alpha$ parameters, there is a significant scatter in the $\alpha$ index values, which can be explained by the inhomogeneous microstructure of the material, as well as by the difference in the direction of propagation of cracks under loading.

\section{CONCLUSIONS}

AE empirical model of a fatigue crack in low-carbon steel 09G2S is presented in the work. As a result of mechanical tests of 53 samples with fatigue cracks under the control of the AE method, a representative sample of data was formed, which allows to correlate the AE parameters and the parameters of the fatigue defect. 
It is shown in the work that the plastic deformation zones at the crack tip have different volumes, even for cracks close in length, which is due to the peculiarities of the metal microstructure near the crack tip. The number of AE impulses emitted by cracks is proportional to the volume of the plastic deformation zone at the crack tip. The number of AE impulses depending on the applied load varies according to a power law, and the power index correlates with the crack length.

\section{ACKNOWLEDGEMENTS}

The work was carried out in the implementation of State Order Project of Ministry of Education and Science of Russian Federation in the field of scientific activity № 11.9879.2017/8.9.

\section{REFERENCES}

1. V. D. Nacik, K. A. Chishko. (1975). The dynamics and sound radiation of a Frank-Reed dislocation source.// Solid state physics.17.342.(inRussian).

2. V. D. Nacik. Sound radiation by a dislocation emerging on the surface of a crystal. JETP Letters, $N$ 8,324.(inRussian).

3. Holt J., Goddart D. J. (1980). Acoustic emission during the Elastic-Plastic Deformation of Low Alloy Reactor Pressure Vessel Steels. II: Deformation around a crack. Material Science and Engineering, V.44, 267-277.

4. BoykoV. S., V. D. Nacik. (1978). Elementary dislocation mechanisms of acoustic emission. Elementary processes of plastic deformation of crystals. Naukovadumka. (in Russian).

5. Merson D. L, et al.(2018). The nature of acoustic emission during deformation processes in metals and alloys. Proceedings of the IXth Eurasian Scientific and Practical Conference. 138. (in Russian).

6. Semashko N. A., atal. (2000). Changing Ti-Al alloy structure during deformation. Promising materials. №1. (in Russian).

7. Bashkov O. V., Semashko N. A. (2004). Acoustic emission with changing deformation mechanisms of plastic structural materials// Physical Mesomechanics, № 6, 59-62.

8. Eneh, A. E. (2015). Application Of Recycled Plastics And Its Composites In The Built Environment. Best International Journal Of Management, Information Technology And Engineering, 3(3), 9-16.

9. Bibik Z. I. (1987). Acoustic emission during deformation of pure single crystals of aluminum. Physics of metals and metal science,№63(4), 811-815. (in Russian).

10. Vinogradov, et al. (1996). Acoustic Emission Analysis of Grain Boundary Effect on Plastic Deformation of Bicrystals. Materials Science Forum, V. 210-213, 565-572.

11. Baram J., Rosen M. (1980). Effect of grain size on the acoustic emission generated during plastic deformation of aluminium. Mater. Science and Eng, V.45, 255-261.

12. Hamstad M. A., et al. (1977). A correlation between acoustic emission and the fracture toughness of 2124-T851 aluminium. Engineering Fracture Mechanics, V. 9, 663-674.

13. Zarmai, M. T., Ekere, N. N., Oduoza, C. F., \& Amalu, E. (2015). Effect of intermetallic compounds on thermo-mechanical reliability of lead-free solder joints in solar cell assembly. International Journal of Mechanical Engineering.

14. Skalskyi V. R., Koval P. M. (2007). Same methodological aspects of application of acoustic emission. Lviv: Publishing House Spolom.

15. Botvina L. R. (2011). Damage evolution on different scale levels. Izvesiya. Physics of the Solid Earth,№ 10, 5-18. 
16. GOST 20295-85. Steel welded pipes for main gas-and-oil pipelines. Specifications.

17. GOST 52910-2008. Vertical cylindrical steel tanks for oil and oil-products. General specifications.

18. PB 03-593-03. Regulations on Organization and Execution for Acoustic-Emission Inspection of Vessels, Boilers, Apparatus and Process Piping

19. Umanath, K., Devika, D., \& Seshabhattur, S. A Review on Abrasive Water Jet Milling of High Strength Materials.

20. Griffith A. (1921). The Phenomena of Rupture and Flow in Solids. Phil. Trans. R. Soc. Lond. A., Vol. 221, 163-198. 18

21. Parton V. Z. (2007). Fracture Mechanics: from theory to practice. CRC Press.

22. Matyunin V. M., et al. (2015). A size effect in the indentation of materials at the micro-and nanoscale strained volumes. Piezoelectrics and Nanomaterials: Fundamentals, Developments and Applications. Nova publishers.

\section{AUTHOR PROFILE}

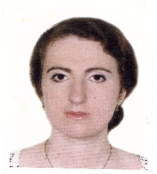

Barat Vera is Associated professor of National Research University (MPEI). Area of professional activity is acoustic emission and signal processing methods. Received a Ph.D. at the National Research Institute of MPEI in 2001, the author more than 70 scientific publication in national and international journals. Member of European working group on acoustic emission (EWGAE).

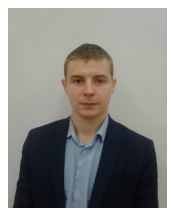

Marchenkov Artem is associated professor of National Research University (MPEI). Area of professional activity is material science and nondestructive testing methods. Received a Ph.D. at the National Research Institute MPEI in 2015, the author more than 90 scientific publication in national and international journals. Expect of University of Shanghai Cooperation Organization (USCO).

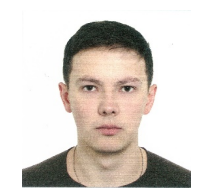

Bardakov Vladimir is senior researcher in INTERUNIS-IT and graduate student of National Research University (MPEI), author of more than 30 scientific publications in national and international journals. Area of professional activity is acoustic emission and technical diagnostics. Currently working on a Ph.D. thesis on acoustic emission testing of concrete structures. 